\title{
Article \\ Assessing Waterlogging Stress Level of Winter Wheat from Hyperspectral Imagery Based on Harmonic Analysis
}

\author{
Feifei Yang ${ }^{1}{ }^{\mathbb{D}}$, Shengping Liu ${ }^{1}$, Qiyuan Wang ${ }^{2}{ }^{\mathbb{D}}$, Tao Liu ${ }^{3}$ and Shijuan $\mathrm{Li}^{1, *}$ \\ 1 Agricultural Information Institute, Chinese Academy of Agricultural Sciences, Beijing 100125, China; \\ yangfeifei@cau.edu.cn (F.Y.); liushengping@caas.cn (S.L.) \\ 2 Institute of Land Reclamation and Ecological Restoration, China University of Mining \& Technology (Beijing), \\ Beijing 100081, China; qiyuanwang@student.cumtb.edu.cn \\ 3 Agricultural College, Yangzhou University, Yangzhou 225009, China; tliu@yzu.edu.cn \\ * Correspondence: lishijuan@caas.cn
}

check for updates

Citation: Yang, F.; Liu, S.; Wang, Q.; Liu, T.; Li, S. Assessing Waterlogging Stress Level of Winter Wheat from Hyperspectral Imagery Based on Harmonic Analysis. Remote Sens. 2022, 14, 122. https://doi.org/ $10.3390 /$ rs 14010122

Academic Editor: Brigitte Leblon

Received: 23 November 2021

Accepted: 25 December 2021

Published: 28 December 2021

Publisher's Note: MDPI stays neutral with regard to jurisdictional claims in published maps and institutional affiliations.

Copyright: (C) 2021 by the authors. Licensee MDPI, Basel, Switzerland. This article is an open access article distributed under the terms and conditions of the Creative Commons Attribution (CC BY) license (https:// creativecommons.org/licenses/by/ $4.0 /)$.
Abstract: Frequent waterlogging disasters can have serious effects on regional ecology, food safety, and socioeconomic sustainable development. Early monitoring of waterlogging stress levels is vital for accurate production input management and reduction of crop production-related risks. In this study, a pot experiment on winter wheat was designed using three varieties and seven gradients of waterlogging stress. Hyperspectral imagery of the winter wheat canopy in the jointing stage, heading stage, flowering stage, filling stage, and maturation stage were measured and then classified. Wavebands of imaging data were screened. Waterlogging stress level was assessed by a combined harmonic analysis method, and application of this method at field scale was discussed preliminarily. Results show that compared to the k-nearest neighbor and support vector machine algorithms, the random forest algorithm is the best batch classification method for hyperspectral imagery of potted winter wheat. It can recognize waterlogging stress well in the wavebands of red absorption valley (RW: 640-680 nm), red-edge (RE: 670-737 nm), and near-infrared (NIR: 700-900 nm). In the RW region, amplitudes of the first three harmonic sub-signals (c1, c2, and c3) can be used as indexes to recognize the waterlogging stress level that each winter wheat variety undertakes. The third harmonic sub-signal amplitude $\mathrm{c} 3$ of the RE region is also suitable for judging stress levels of JM31 (one of the three varieties which is highly sensitive to water content). This study has important theoretical significance and practical application values related to the accurate control of waterlogging stress, and functions as a new method to monitor other types of environmental stress levels such as drought stress, freezing stress, and high-temperature stress levels.

Keywords: waterlogging stress level; hyperspectral imagery; harmonic analysis; winter wheat

\section{Introduction}

Agricultural production is strongly dependent on climate conditions. As one of the major abiotic stresses, waterlogging disasters are becoming a dominant constraint on crop growth. There are reports of waterlogging disasters around the world, such as in Egypt and Saudi Arabia [1], Ethiopia [2], Bihar [3] and the Gangetic Plains [4], India, the Yangtze River Delta, and across China [5] (including Jiangsu Province, Zhejiang Province, Anhui Province, and Shanghai City). A rice-wheat rotation system is the dominant cultivation mode in the agricultural district of the Yangtze River Delta, in which it accounts for $20 \%$ of total wheat sown area and $22 \%$ of total wheat grain yield in China [6]. Waterlogging disasters can affect stomatal conductance, hydraulic conductivity, transpiration, respiration, photosynthesis rates, yield, and metabolism rates of overground parts of crops [7-9]. Frequent occurrences of waterlogging disasters can significantly influence regional ecology, food safety, and socioeconomic sustainable development. The early monitoring of waterlogging stress levels is vital to accurate production input management (e.g., of water, fertilizer, and pesticide) and reduction of crop production-related risks. Providing information about crop 
status as early as possible is of important significance to formulating policies concerning price, circulation, and storage of foods [10]. Traditional monitoring methods are lacking in several ways, including that they are time-consuming, and that macroscopic monitoring is not possible. Hyperspectral remote sensing is information-rich and nondestructive and can realize the monitoring of waterlogging stress in real time. The real-time monitoring of crops waterlogging stress levels based on hyperspectral remote sensing information is significant to the timely implementation of antidisaster and production resumption technologies.

At present, studies on monitoring the environmental stress levels of crops based on hyperspectral remote sensing have mainly focused on disease stress levels and the presence of heavy metals. To detect the heavy metal stress level of vegetation, Hede et al. [11] built a new vegetation index considering greenness and shortwave infrared (VIGS) and found that VIGS was superior to normalized difference vegetation index (NDVI) in detecting vegetation stress level. Liu et al. [12] built a characteristic space for heavy metal stress monitoring based on the phenological index and dry weight of roots (WRT) by using the time-series image set of NDVI and WOFOST models. This space could recognize the heavy metal stress level of rice effectively with an accuracy of greater than 95\%. Zhang et al. [13] found that the average value (AVG) and standard deviation (SD) of canopy-air temperature difference (Tc-Ta) were relatively good indexes to determine the heavy metal stress level of rice. Li et al. [14] combined hyperspectral and radar remote sensing to monitor rice and found that NVI (R598, R508) of the canopy chlorophyll index and SVI (HV, VH, HH, and $\mathrm{VV}$ ) of the microwave index were sensitive to changes of chlorophyll content and biomass under heavy metal stresses and constructed a two-dimensional feature space model of heavy metal stress. Based on the guided regularized random forest (GRRF), Dhau et al. [15] screened the best wavebands to recognize different levels of maize streak, which were $552 \mathrm{~nm}, 603 \mathrm{~nm}, 683 \mathrm{~nm}, 881 \mathrm{~nm}$, and $2338 \mathrm{~nm}$, respectively. Qiao et al. [16] built a take-all disease-grade prediction model of wheat based on the support vector machine (SVM) and found that the best training effect was achieved when choosing the 700-900 nm waveband. Jin et al. [17] found that the recognition model of the verticillium wilt severity in cotton based on the combination of wavelet transform and SVM algorithm was the best. Huang et al. [18] proposed the wheat scab index (WSI) by choosing the sensitive wavebands 450-488 $\mathrm{nm}$ and 500-540 nm, based on which a reversion model of wheat scab severity was built up. Some studies have focused on monitoring freezing and dust stress levels. Zhang et al. [19] discussed the possibility of maize seed classification under different freezing stress levels by combining hyperspectral images and deep convolutional neural networks (DCNN), finding that the model performed positively. Liang et al. [20] applied the random forest (RF) algorithm to establish a hyperspectral diagnosis model based on dust stress level by using the dust stress normalized index (DSNI) as the input parameter, which achieved a prediction accuracy of $87.5 \%$.

Existing studies concerning the monitoring of waterlogging stress based on hyperspectral remote sensing have focused primarily on monitoring a single occurrence of waterlogging stress and distinguishing between two environmental stresses. Regarding the former, Yang et al. [21] established a rapid determination model of leaf water content for monitoring waterlogging in winter wheat, combined with methods such as vegetation index construction and BP neural network based on hyperspectral parameters. Zhao et al. [22] detected waterlogging stress of cotton based on hyperspectral images and convolutional neural network. Xiong et al. [23] were able to reflect waterlogging stress in wheat by the mean difference of spectra in the 670-2400 nm waveband. Regarding the latter, Jiang et al. [24] discovered that AREAred/AREAgreen and AREAred*AREAgreen can effectively distinguish vegetation under $\mathrm{CO}_{2}$ leakage and waterlogging stress. Emengini et al. [25] proved that vegetation under petroleum pollution, waterlogging, and oil stressors might be distinguished by hyperspectral and thermal infrared remote sensing. There are currently some studies on assessing waterlogging stress levels of vegetation. Xia et al. [26] detected waterlogging stress levels combined with classification algorithms based on hyperspectral images of oilseed rape leaves. Yang et al. [27] evaluated waterlogging stress levels of 
winter wheat combined with spectral difference entropy based on the leaf and canopy hyperspectral data. However, the research is still insufficient, and the main reasons include, firstly, that different levels of waterlogging stress are difficult to control due to the influences of farmland evapotranspiration, precipitation, and runoffs, while pot experiments are implemented under controllable environmental conditions and are repeatable and reproducible [28,29]. Secondly, different levels of waterlogging stress have little difference in the effect of vegetation spectrum. Harmonic analysis is often applied to signal processing of electrical systems [30]. In the field of remote sensing, harmonic analysis has achieved good results in land cover classification [31], time series analysis [32], etc. Harmonic analysis has a strong ability to analyze signals. Harmonic decomposition of the spectrum can decompose weak spectral distortion containing environmental stress in the vegetation spectrum into low-order harmonics and decompose high-frequency noise into high-order harmonics. This is more beneficial for analyzing useful information in the spectral signals [33]. Spectral signals of vegetation can be viewed as superposition of a series of sub-signals with different frequencies, phases, and amplitudes after harmonic decomposition. Under waterlogging stress, the reflection spectra of vegetation might change in the spectral space and the corresponding sub-signals will also change. The weak spectral distortion of vegetation, which is caused by different levels of waterlogging stress, might be easier to detect in the harmonic characteristic space, and analysis methods in the frequency domain may be easier for realizing the feature extraction of weak information on vegetation under environment stress.

In this study, we assessed the waterlogging stress levels of winter wheat from the hyperspectral imagery by a combined harmonic analysis method through pot and field experiments (the experiments were set up in Yangzhou City, Jiangsu Province, China, which was prone to waterlogging disasters). The specific objectives of this study were (1) to compare the batch classification accuracy of RF, SVM, and k-nearest neighbor (KNN) algorithms for potted hyperspectral images, to extract the spectral data of winter wheat after classification more accurately; (2) to introduce a spectral distance $\left(d_{i}\right)$ to quantitatively evaluate the capacities of different spectra to recognize winter wheat under waterlogging stress in order to select the wavebands with strong recognition ability; (3) to develop a harmonic analysis method to assess the waterlogging stress levels in a frequency domain different from common spectral dimensions. This study has representativeness and lays the foundations for large-scale monitoring of crops after waterlogging disasters.

\section{Materials and Methods}

\subsection{Experimental Design}

The experiments were carried out in Yangzhou City $\left(119^{\circ} 25^{\prime} \mathrm{N}, 32^{\circ} 23^{\prime} \mathrm{E}\right)$, Jiangsu Province, China between 2018 and 2019 (Figure 1). Yangzhou City is in the center of Jiangsu Province and is a transitional region between subtropical monsoon and temperate monsoon climates. There are four distinct seasons, sufficient sunshine, and adequate precipitation. Most precipitation falls from March to May, which is the growth season of wheat. Moreover, there are flat terrains, rich water systems, and viscous soils, which are easily affected by waterlogging disasters.

Pot experiment: The pot experiment was carried out in the awning of the potting experiment field in Yangzhou University, Yangzhou City. Seeds of winter wheat were sown on 10 November 2018 at a density of 2 seeds per hole, 8 holes per pot. In three leaves and one half-leaf period, seedlings were singled to 8 per pot. Wheat was harvested on 28 May 2019. The inner diameter at the bottom of the pot, inner diameter at the mouth, height, and net pot weight were $20 \mathrm{~cm}, 28 \mathrm{~cm}, 29 \mathrm{~cm}$, and $0.54 \mathrm{~kg}$, respectively. Each pot was filled with $10 \mathrm{~kg}$ light dried soils and $5.28 \mathrm{~g}$ compound fertilizer which was made up of $\mathrm{N}-\mathrm{P}-\mathrm{K}$ at a ratio of $15-15-15 \%$. Another $1 \mathrm{~kg}$ of soil was used for cover after sowing and $3.52 \mathrm{~g}$ compound fertilizer was applied in the jointing stage. Water control was conducted by weight and began when the winter wheat reached jointing stage (15 March 2019) to implement the gradient tests of waterlogging stress. All waterlogging stresses were ended 
on 30 March 2019. Subsequently, equal volumes of water were provided for all pots until maturation and harvest. Three test factors were set: waterlogging stress levels (control CK, mild waterlogging ML, and severe waterlogging SL) in the jointing stage, stress duration ( $5 \mathrm{~d}, 10 \mathrm{~d}$, and $15 \mathrm{~d}$ ), and wheat varieties (YF4, JM31 and JM38). Specifically, the relative soil water contents of CK and ML were controlled at 70-80\% and 85-90\%, while the SL retained about $1.5 \mathrm{~cm}$ of water on the surface of the soil. Among them, YF4 was the normal variety, while JM31 was extremely sensitive to water, and JM38 was extremely insensitive to water. A total of 21 treatment groups were set, and each treatment had 9 repetitions, with a total of 189 pots. Eight wheat samples were provided per pot, and 1512 wheat samples were provided for the test (Figure 2).

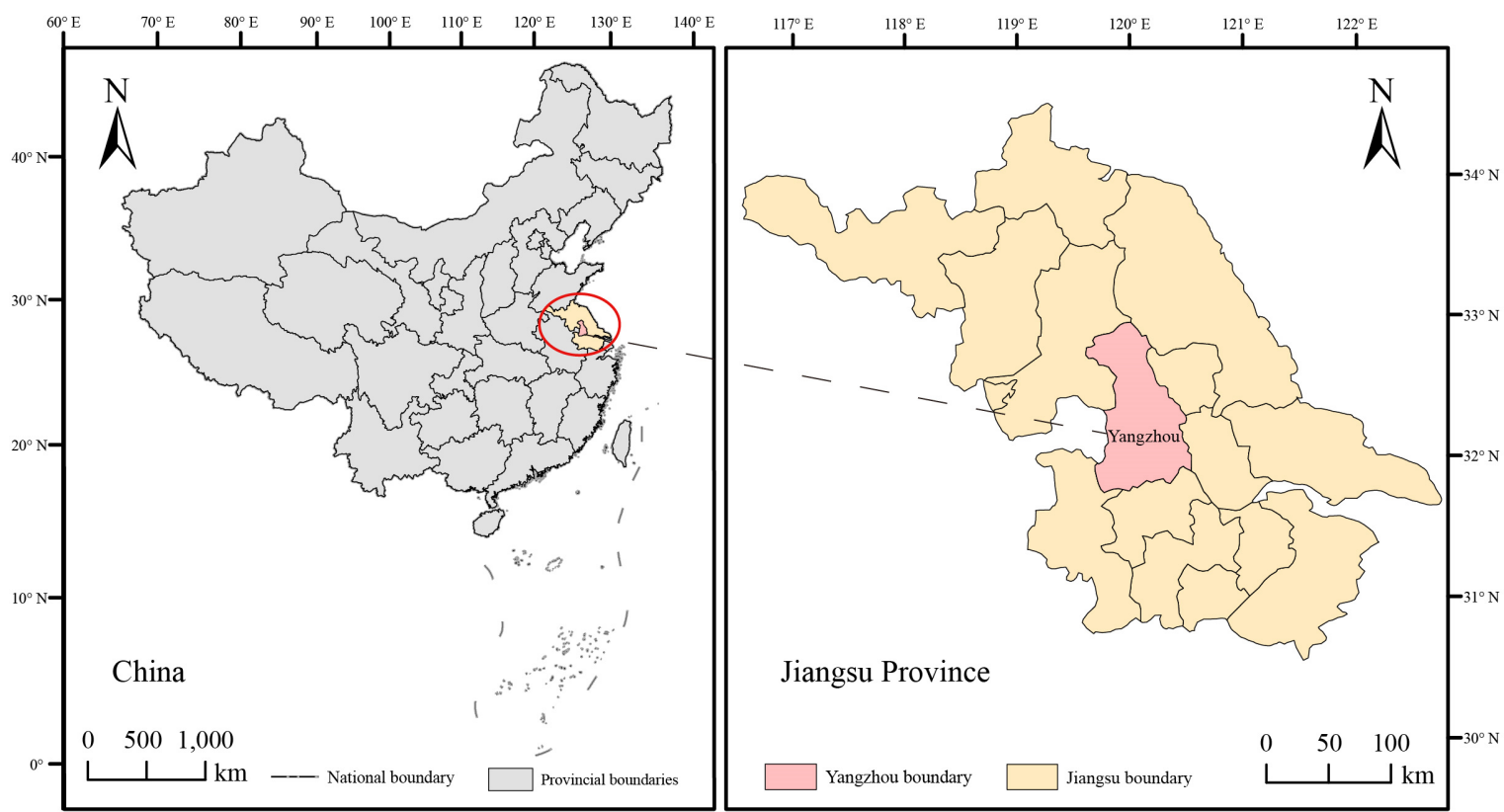

Figure 1. Location of test area.

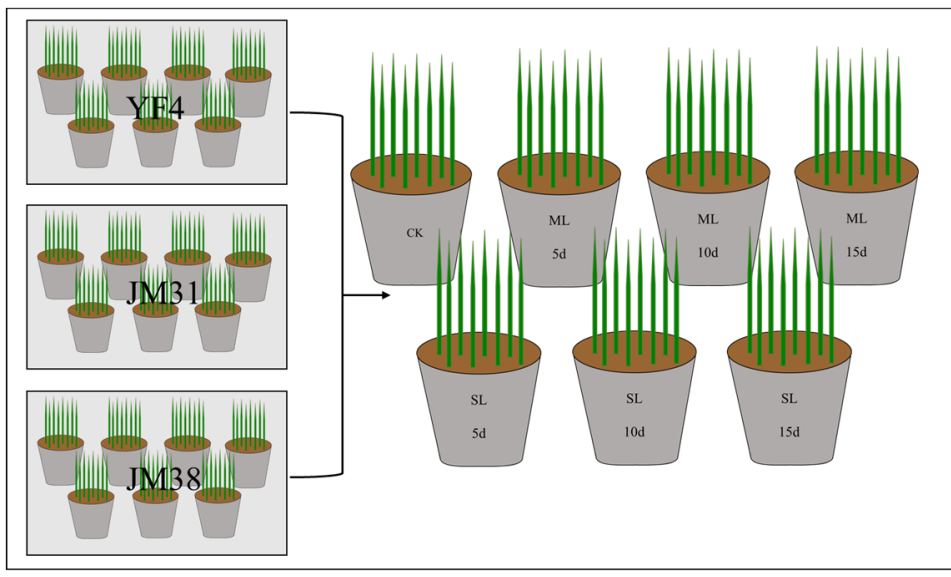

(a)

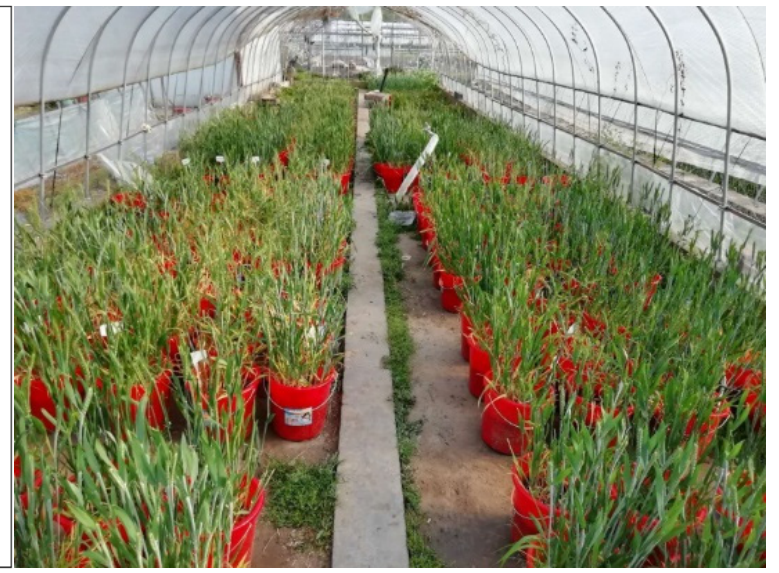

(b)

Figure 2. Pot experiment of winter wheat. (a) Schematic diagram of experimental design; (b) pot experiment site.

Field experiment: The field experiment was carried out in Yizheng modern agricultural demonstration base, Yangzhou City. Seeds were sown on 4 November 2018, and wheat was harvested on 5 June 2019. Cultivation measures are the same as those of local high-yield fields. Two treatments (Figure 3; blue box-control, red box-waterlogging stress) were set 
for 30 varieties of winter wheat. The waterlogging stress treatment began when the winter wheat reached the jointing stage (12 March 2019), maintaining the water layer about $1.5 \mathrm{~cm}$ for 10 days. Subsequently, all plots were planted normally until maturation and harvest.

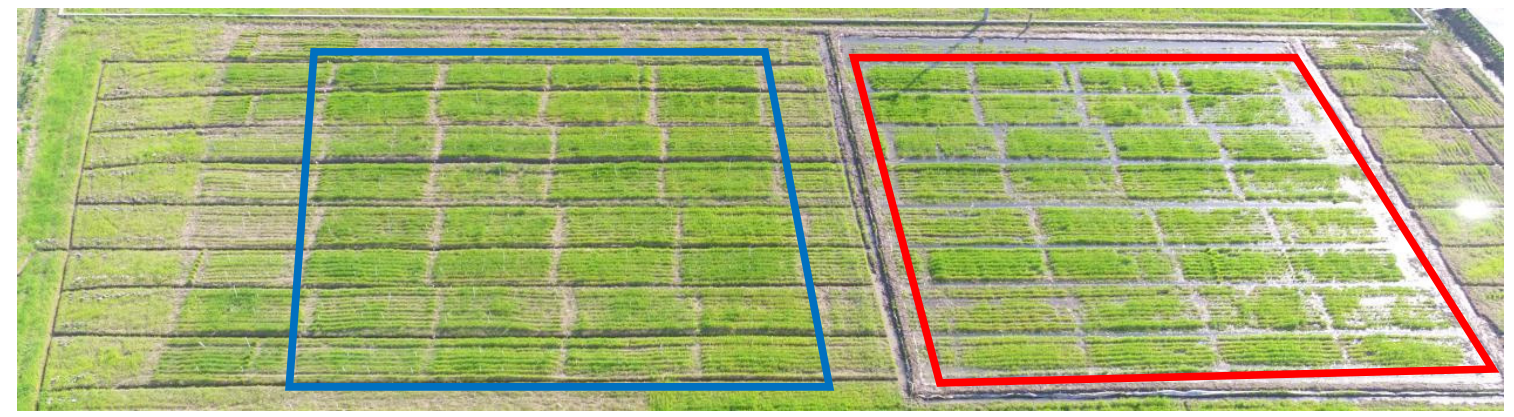

Figure 3. Field experiment of winter wheat. Blue box—control; red box-waterlogging stress.

\subsection{Canopy Hyperspectral Imagery Acquisition}

Proximally-sensed hyperspectral imagery: The proximally-sensed hyperspectral imagery of the potted winter wheat was measured using the Gaiasky-mini2 imaging spectrometer (Sichuan Shuanglihepu Technology Co., Ltd., Chengdu, China; spectral region: 400-1000 nm; sampling interval: $3.5 \mathrm{~nm} \pm 0.5 \mathrm{~nm}$ ). The Gaiasky-mini2 imaging spectrometer has the functions of automatic exposure and automatic scanning speed matching. Before each acquisition, the focus was adjusted in advance. The method was roughly to place the target $1 \mathrm{~m}$ away from the lens to adjust the imagery to clarity, and a standard white plate was used for calibration before measurements. The data was collected under illumination conditions from 10:00 to 14:00 on sunny days. Observations were made on five pots per group. Measurements were taken every $7 \mathrm{~d}$ since the day of waterlogging in the jointing stage until commencement of the maturation stage, excluding cloudy and rainy days. When collecting data, the Gaiasky-mini2 imaging spectrometer was supported by a tripod with its lens downward. It was situated $1 \mathrm{~m}$ away from the wheat canopy to take pictures (Figure 4a).

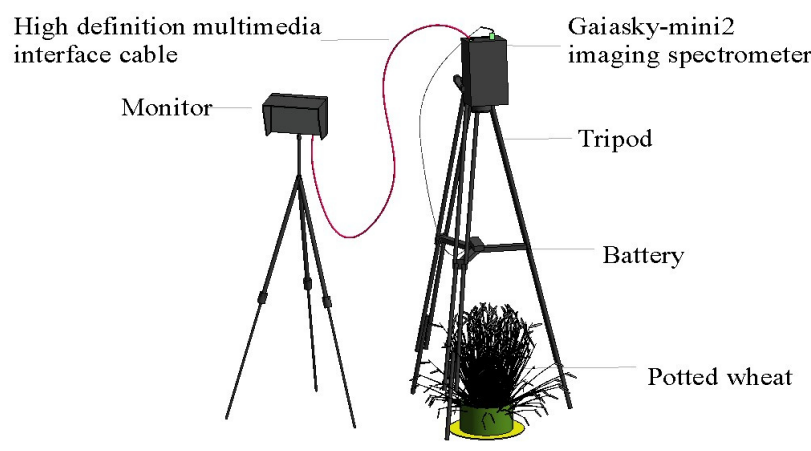

(a)

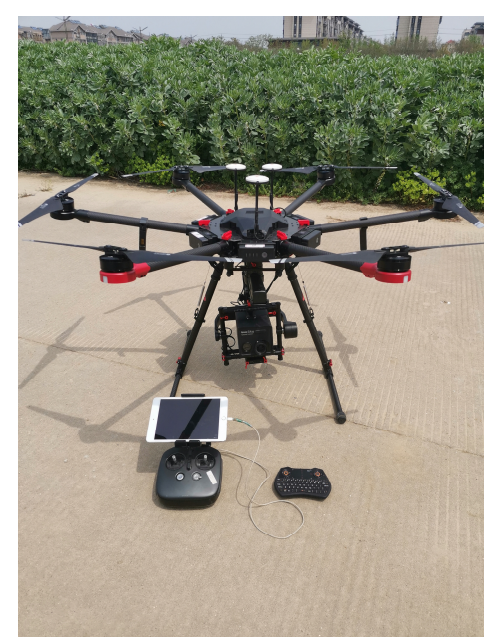

(b)

Figure 4. Canopy hyperspectral imagery acquisition. (a) Proximally-sensed hyperspectral imagery; (b) UAV hyperspectral imagery.

UAV hyperspectral imagery: The UAV hyperspectral imagery of the field winter wheat was captured by the DJI M600 UAV (SZ DJI Technology Co., Ltd., Shenzhen, China) equipped with the Gaiasky-mini2 imaging spectrometer. The focus was adjusted in advance to make the imagery clear when the distance to the target is infinite, and a standard white 
plate was used for calibration before measurements. The data was collected from 10:00 to 14:00 on a sunny day in the heading stage of winter wheat. Automatic shooting occurred on the planned route, the flight altitude was set to $50 \mathrm{~m}$, the speed was $3 \mathrm{~m} / \mathrm{s}$, and the fore-and-aft overlap and side overlap were $60 \%$ and $50 \%$, respectively (Figure $4 \mathrm{~b}$ ).

\subsection{Data Processing}

\subsubsection{Preprocessing}

Preprocessing of the hyperspectral imagery includes lens correction, reflectance correction, atmospheric correction, geometric calibration, and image stitching. The lens correction can eliminate the distortion and brightness unevenness in the data acquisition process. The imaging of the lens edge and the lens center can be kept consistent after lens correction. The reflectance correction in SpecView includes radiometric calibration and reflectance value output. It can establish the quantitative relations between digital number (DN), which is the output of each probe's elements in the imaging spectrometer, and the output radiance and reflectance value in the corresponding field of view. Atmospheric correction can eliminate the influences of atmosphere, illumination, and other environmental factors on the electromagnetic radiation level of surface features, ensuring that measurements correspond with the real reflectivity of surface features. Geometric calibration can eliminate geometric distortion on images and transform it onto the reference image coordinate system. SpecView can be used for lens correction, reflectance correction, and atmospheric correction, and HiSpectralStitcher can be used for geometric calibration and image stitching (Sichuan Shuanglihepu Technology Co., Ltd., Chengdu, China).

The proximally-sensed hyperspectral imagery acquisition is hardly affected by the atmospheric and geometric distortion, so these data only need lens correction and reflectance correction. The preprocessing of UAV hyperspectral imagery includes all the above steps.

\subsubsection{Image Classification}

\section{- Classification algorithm}

RF [34] is an integrated classifier model which is composed of many decision tree classification models. It receives several training subsets from the original training set through bootstrap sampling, and then implements the node splitting decision-making tree modeling to each subset by using an algorithm. Several decision trees are combined into a random forest, and results are determined through prediction votes from every tree. SVM $[35,36]$ is a supervised parameter-free statistical learning method based on VC-dimensional theory and minimum structural risks. It seeks the optimal values between model complexity and learning capacity under the limited sample information. KNN [37] believes that testing sample points belong to the nearest point set. It firstly calculates the distance or similarity between the testing samples and training samples of known categories, then identifies the $\mathrm{K}$ neighbors which are nearest to the classification sample data in distance or similarity. Secondly, sample data are classified according to the classes of these neighbors.

- Accuracy evaluation

Classification accuracy was evaluated by visual interpretation and classification accuracy based on the confusion matrix. The former method of evaluation is not introduced in the present study. Confusion matrices, also known as error matrices, are mainly used to analyze differences between classification results and true values. They can display classification results in one matrix, thus allowing for the assessment of various accuracies, including overall accuracy (OCA), user accuracy (UA), producer accuracy (PA), and Kappa coefficient.

\subsubsection{Waveband Selection}

During waveband selection, wavebands which have greater interclass dispersion and less intraclass dispersion are expected to be chosen. According to the Fisher criterion, the 
relative distance among different classes can be used as measurement of class separability. Moreover, the separability is larger when the relative distance is longer [38]. For quantitative assessment of the capacity of spectra in different wavebands to recognize waterlogging stress in winter wheat, a spectral distance $\left(d_{i}\right)$ was introduced, representing the relative spectral distance on the $i$ th waveband between stressed and control winter wheat. Next, the mean of spectral distance $\left(D_{\mu}\right)$, maximum $\left(D_{\max }\right)$, minimum $\left(D_{\min }\right)$, and coefficient of variation $\left(D_{c v}\right)$ [39] were used to judge capacity for recognition of a spectral range. These parameters are defined as follows:

$$
\begin{gathered}
d_{i}=\left|X_{i}-Y_{i}\right| \\
D_{\mu}=\frac{1}{n} \sum_{i=1}^{n} d_{i} \\
D_{c v}=D_{\sigma} / D_{\mu} \\
D_{\max }=\max \left(d_{1}, d_{2}, \ldots, d_{i}, \ldots, d_{n}\right) \\
D_{\min }=\min \left(d_{1}, d_{2}, \ldots, d_{i}, \ldots, d_{n}\right)
\end{gathered}
$$

where $X_{i}$ and $Y_{i}$ are spectral values of stressed and control winter wheat on the $i^{\text {th }}$ waveband, respectively; $n$ is the number of wavebands in a spectral range; $D_{\sigma}$ and $D_{\mu}$ are the mean and standard deviation of spectral distance in this spectral range, respectively.

\subsubsection{Harmonic Analysis}

Harmonic analysis is a basic method of signal processing. The method of expressing time series by the sine (cosine) harmonic superposition is called harmonic analysis (HA). It transforms the time series from the time domain to the frequency domain and finds the transformation parameters. In the frequency domain, the time domain component is represented by the superposition of multiple sine (co)sine curves with different frequencies [40]. In this study, the original spectral signal can be decomposed into a series of combined sine and cosine waves with different amplitudes and phases. Features of different components of the original spectral signal can be analyzed in the frequency domain. The formulas are as follows [41]:

$$
\begin{gathered}
V(x)=\frac{A_{0}}{2}+\sum_{h=1}^{\infty}\left[A_{h} \cos (2 h \pi x / N)+B_{h} \sin (2 h \pi x / N)\right] \\
=\frac{A_{0}}{2}+\sum_{h=1}^{\infty}\left[C_{h} \sin \left(2 h \pi x / N+\varphi_{h}\right)\right]
\end{gathered}
$$

where:

$$
\begin{gathered}
\frac{A_{0}}{2}=\frac{1}{N} \sum_{k=1}^{N} v_{k} \\
A_{h}=\frac{2}{N}\left(v_{1} \cos \frac{2 \pi h}{N}+v_{2} \cos \frac{4 \pi h}{N}+\ldots+v_{N} \cos 2 \pi h\right) \\
B_{h}=\frac{2}{N}\left(v_{1} \sin \frac{2 \pi h}{N}+v_{2} \sin \frac{4 \pi h}{N}+\ldots+v_{N} \sin 2 \pi h\right) \\
C_{h}=\sqrt{A_{h}^{2}+B_{h}^{2}} \\
\varphi_{h}=\arctan \left(A_{h} / B_{h}\right)
\end{gathered}
$$

where $V(x)$ is the signal being analyzed; $N$ is the total number of wavebands; $\frac{A_{0}}{2}$ is the harmonic remainder; $A_{h}, B_{h}$, and $C_{h}$ are cosine amplitude, sine amplitude, and harmonic component amplitude at the $h$ th harmonic decomposition, respectively; $\varphi_{h}$ is the initial phase of the $h$ th harmonic decomposition; and $v_{k}$ is the reflectivity of the $k$ th waveband in the reflection spectra. 
In this study, preprocessing was performed based on SpecView and HiSpectralStitcher. Image classification, waveband selection and harmonic analysis were performed based on ENVI5.3, MATLAB R2017a, and Origin 2017.

\section{Results}

\subsection{Image Classification}

The batch classification results of RF, SVM, and KNN were compared. Accuracy was evaluated by visual interpretation and confusion matrix. The classification algorithm with the highest accuracy was chosen for batch classification of 504 hyperspectral images. The number of decision trees (ntree) of RF was set to 10. As there are many images for classification, one representative image (Figure 5) was chosen for better comparison of the three algorithms. According to visual analysis, many winter wheat images were missed in SVM and KNN, showing evidence of the "spiced salt phenomenon". RF is the best algorithm, and it hardly misses images during classification. The classification results of RF conform to practical situations.

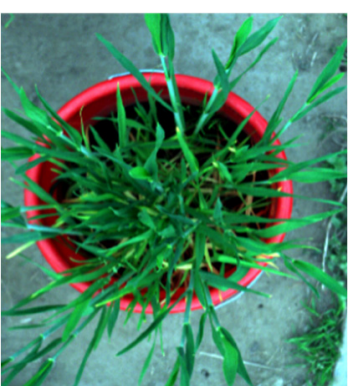

Winter wheat

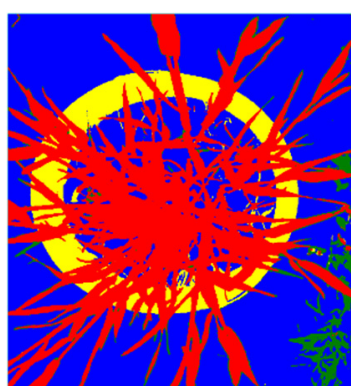

Pot

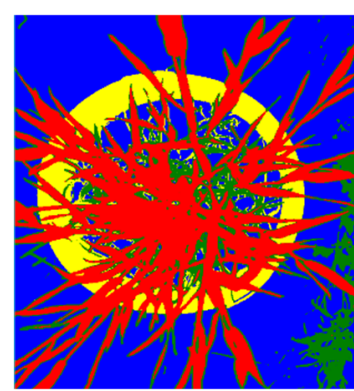

Grass

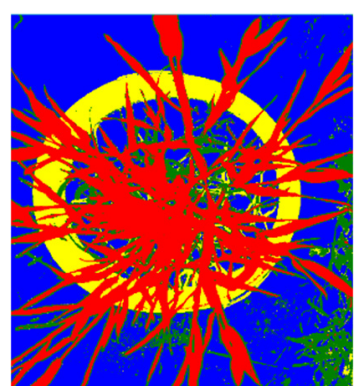

(d)

(b)

Figure 5. Comparison of classification results of RF, SVM, and KNN algorithms. (a) Original image; (b) RF; (c) SVM; (d) KNN.

Confusion matrices were calculated for the classification results of RF, SVM, and KNN. The performance of these three algorithms was evaluated based on their OCA, PA, UA, and Kappa coefficients. Results are shown in Table 1. The RF algorithm showed the highest OCA, which was 3.39\% and 7.61\% higher than that of the SVM and KNN algorithms, respectively. Moreover, the RF algorithm also presented the highest classification accuracy for different classes. For winter wheat, the PA of the RF algorithm was 1.17\% and 1.01\% higher than that of the SVM and KNN algorithms, and its UA was $1.76 \%$ and $3.53 \%$ higher than that of the SVM and KNN algorithms, respectively. With respect to land cover types, the classification accuracy of grass (Aegilops tauschii Coss., Alopecurus aequalis Sobol., etc.) was increased the most. The PA of the RF algorithm was $6.41 \%$ and $8.22 \%$ higher, and UA was $8.55 \%$ and $25.78 \%$ higher compared to that of the SVM and KNN algorithms, respectively. Overall, RF clearly showed the best classification results. Therefore, the RF algorithm was chosen to classify 504 hyperspectral images of potted winter wheat. 
Table 1. Comparison of classification accuracies of RF, SVM, and KNN algorithms.

\begin{tabular}{|c|c|c|c|c|c|c|}
\hline \multirow{2}{*}{ Land Cover Type } & \multicolumn{2}{|c|}{ RF } & \multicolumn{2}{|c|}{ SVM } & \multicolumn{2}{|c|}{ KNN } \\
\hline & PA/\% 1 & UA/ $\% 1$ & PA/\% & UA/\% & PA/\% & UA/\% \\
\hline Winter wheat & 94.89 & 95.89 & 93.72 & 94.13 & 93.88 & 92.36 \\
\hline Pot & 94.62 & 95.84 & 94.31 & 94.31 & 90.98 & 93.65 \\
\hline Soil & 95.21 & 94.45 & 94.59 & 93.28 & 80.29 & 91.23 \\
\hline Grass $^{2}$ & 93.31 & 93.72 & 86.90 & 85.17 & 85.09 & 67.94 \\
\hline OCA $/ \%{ }^{1}$ & \multicolumn{2}{|c|}{95.86} & \multicolumn{2}{|c|}{92.47} & \multicolumn{2}{|c|}{88.25} \\
\hline Kappa coefficient & \multicolumn{2}{|c|}{0.9438} & \multicolumn{2}{|c|}{0.9022} & \multicolumn{2}{|c|}{0.8376} \\
\hline
\end{tabular}

${ }^{1}$ OCA—overall accuracy; PA—producer's accuracy; UA-user's accuracy. ${ }^{2}$ Grass (Aegilops tauschii Coss., Alopecurus aequalis Sobol., etc.).

\subsection{Waveband Selection}

Spectral data of winter wheat after classification was extracted. According to previous studies [42,43], the following waveband ranges were selected for analysis: blue edge (BE, 490-530 nm), green region (GR, 510-560 nm), yellow edge (YE, 550-582 nm), red absorption valley (RW, 640-680 nm), red edge (RE, 670-737 nm), and near-infrared region (NIR, 700-900 nm). For quantitative assessment of the capacities of different spectra to recognize winter wheat under waterlogging stress, a spectral distance $\left(d_{i}\right)$ was introduced. Later, the mean of spectral distance $\left(D_{\mu}\right)$, coefficient of variation $\left(D_{c v}\right)$, maximum $\left(D_{\max }\right)$, and minimum $\left(D_{\min }\right)$ were used to judge recognition capacity of a spectral range. Results are shown in Table 2.

Table 2. Evaluation parameters for waveband selection.

\begin{tabular}{ccccccc}
\hline Evaluation Index & BE & GR & YE & RW & RE & NIR \\
\hline$D_{\mu}$ & 0.018 & 0.021 & 0.025 & 0.033 & 0.050 & 0.085 \\
$D_{c v}$ & 0.111 & 0.106 & 0.034 & 0.010 & 0.294 & 0.156 \\
$D_{\max }$ & 0.021 & 0.024 & 0.027 & 0.033 & 0.077 & 0.098 \\
$D_{\min }$ & 0.015 & 0.017 & 0.024 & 0.032 & 0.032 & 0.045 \\
\hline
\end{tabular}

When the spectra change due to stress, the information size of the corresponding waveband might also change, accompanied by changes in spectral distance between stressed and control winter wheat. As the mean of spectral distance in one waveband range, $D_{\mu}$ is the most direct index to measure recognition ability. A higher value of $D_{\mu}$ indicates a longer spectral distance between the stressed and control winter wheat in this waveband range. As a result, the recognition capacity is higher. $D_{c v}$ was used to measure intraclass dispersion in different waveband ranges and is the ratio between standard deviation and mean of spectral distance in this waveband range. A smaller value of $D_{c v}$ indicates less intraclass variation [39]. As demonstrated in Table $2, D_{\mu}$ reached a maximum in the waveband ranges of NIR, RE, and RW, with values of $0.085,0.050$, and 0.033 , respectively. $D_{c v}$ reached a minimum in the RW, YE, and GR regions, with values of $0.010,0.034$, and 0.010 , respectively. In the RW region, $D_{\mu}$ was relatively high and $D_{c v}$ was relatively low, indicating this range's strong capacity to recognize stressed winter wheat. The $D_{\mu}$ of the RE and NIR regions was relatively large, and although $D_{c v}$ was large as well, the $D_{\min }$ of these regions were higher than the $D_{\max }$ of $\mathrm{BE}, \mathrm{GR}$, and YE. This reflects the fact that the ability of different wavebands to recognize stressed winter wheat varies greatly. The RW (640-680 nm), RE $(670-737 \mathrm{~nm})$, and NIR $(700-900 \mathrm{~nm})$ regions have strong recognition ability, and thus were chosen for harmonic analysis.

\subsection{Harmonic Analysis}

Canopy spectra of winter wheat can be viewed as the superposition of sub-signals with different amplitudes, frequencies, and phases. Different levels of waterlogging stress show different frequencies, phases, and amplitudes in the frequency domain. In this study, 
three harmonic decompositions of canopy spectral data were performed to collect the harmonic remainder, amplitude, and phase of sub-frequency signals in the RW, RE, and NIR regions. It was found that in the RW region, the amplitude of the first harmonic subsignals presented obvious variation laws in the heading stage, filling stage, and flowering stage, but showed no laws in the jointing stage and maturation stage. In this section, the YF4 variety of winter wheat was taken as the example, as the variation trends of the three varieties were similar. The amplitudes of harmonic sub-signals under different waterlogging stress levels in the heading stage, flowering stage, and filling stage within the RW, RE, and NIR regions are shown in Figure 6. The harmonic sub-signals of the RW region are also shown in Figure 7 for further analysis.
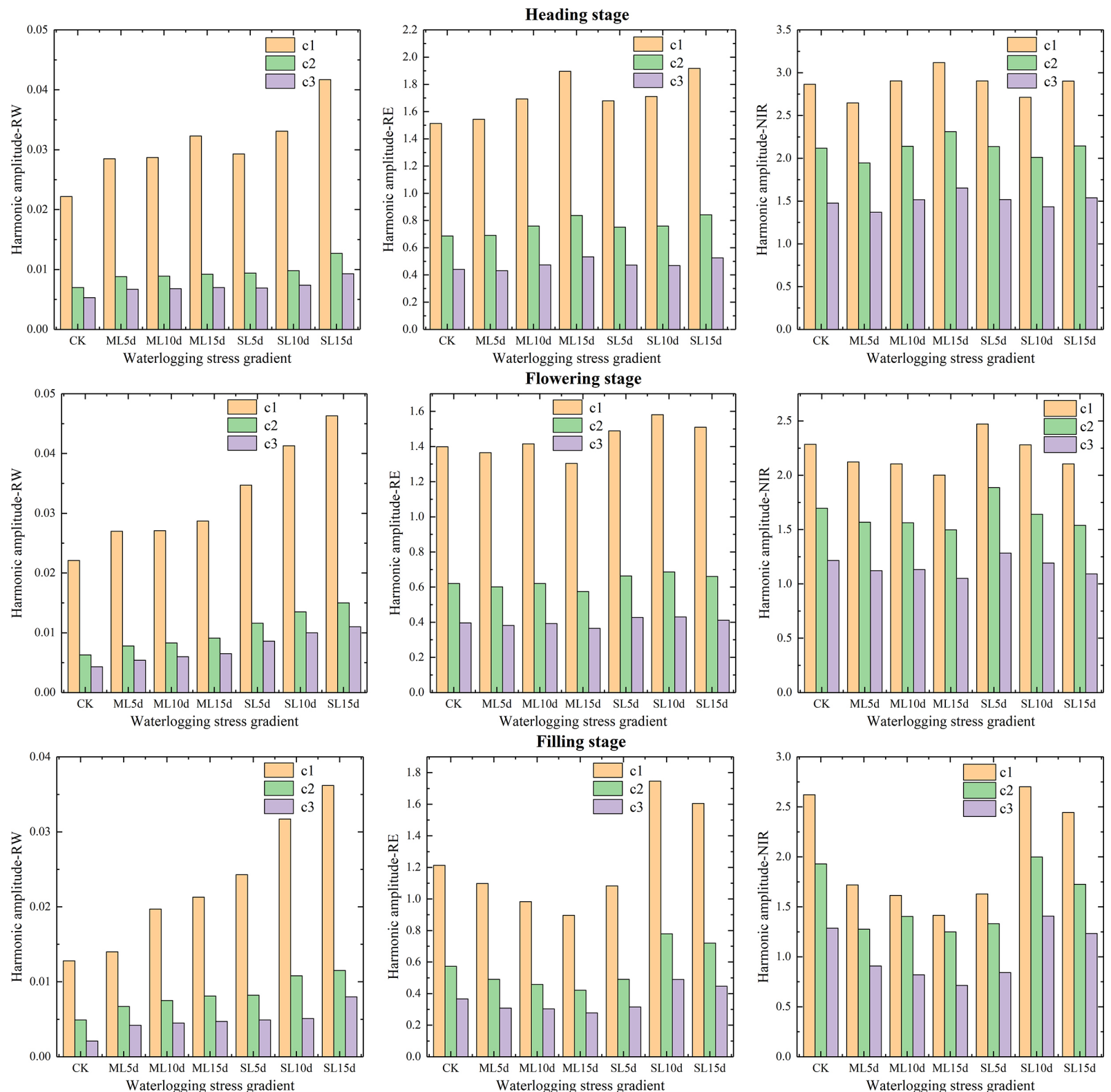

Figure 6. Harmonic amplitudes in the RW, RE, and NIR regions-YF4. 

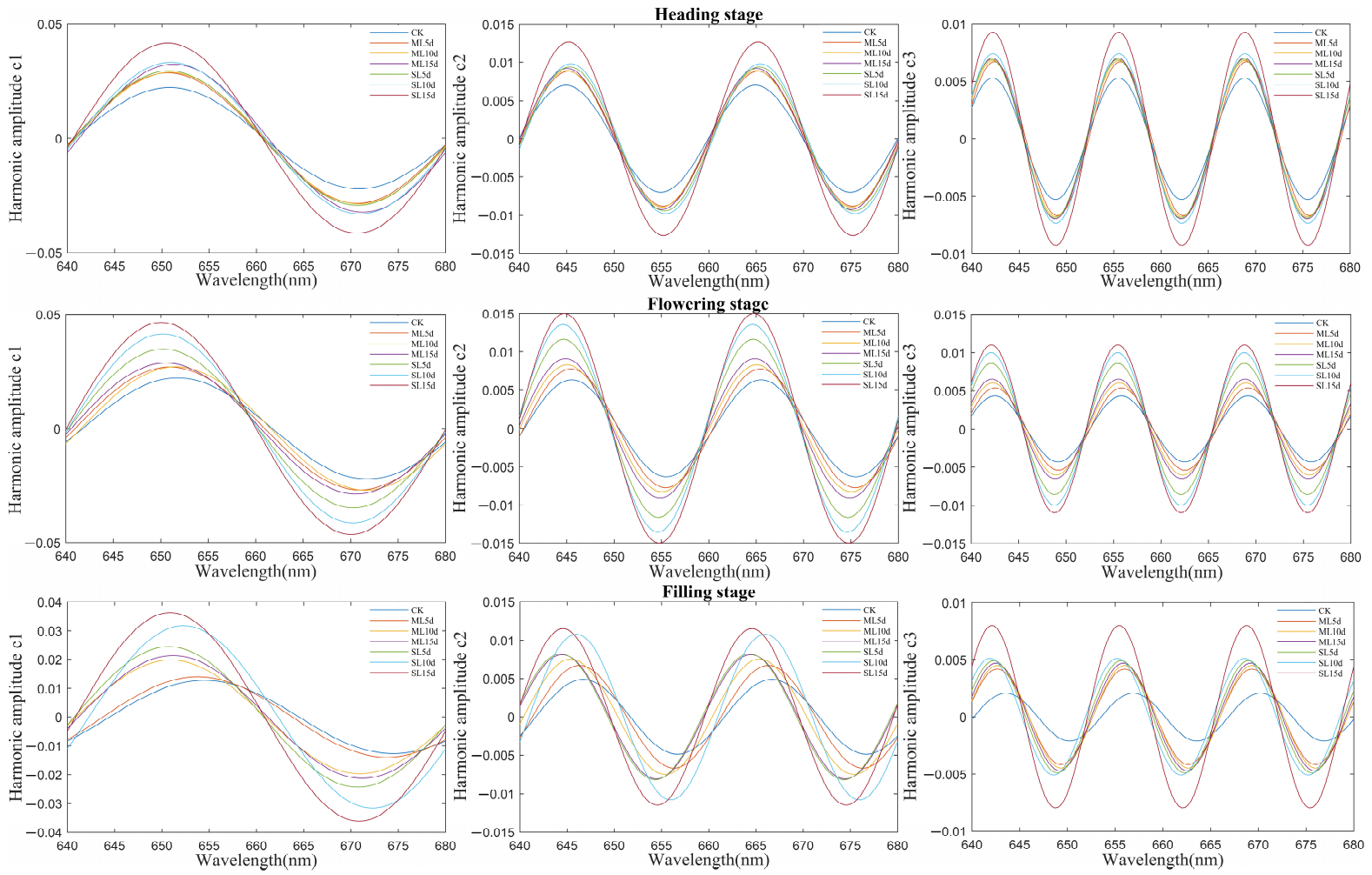

Figure 7. Harmonic sub-signals in the RW region-YF4.

Generally speaking, when the relative soil water contents are the same, the waterlogging stress level increases with the increase of the stress duration; when the stress duration is the same, the stress level increases with the increase of the relative soil water content. That is, the stress level: ML5d < ML10d < ML15d, SL5d < SL10d < SL15d; ML5d < SL5d, ML10d $<$ SL10d, ML15d $<$ SL15d. Combined with Figures 6 and 7, it was found that after harmonic decomposition in the RW region in the heading, flowering, and filling stages, the amplitudes of sub-signals (c1, c2, and c3) increased with an increase in stress levels. In the RE region, the amplitudes of the first two harmonic decompositions in the heading stage were positively correlated to stress levels but presented no evident laws in the follow-up stages. In the NIR region, the amplitude of sub-signals had no evident laws. Therefore, sub-signals $\mathrm{c} 1, \mathrm{c} 2$, and $\mathrm{c} 3$ in the RW region in the heading, flowering, and filling stages can be used to assess waterlogging stress levels. The numerical values of $c 1, c 2$, and c3 were positively correlated with waterlogging stress levels. However, c1, c2, and c3 in the RE and NIR regions could not be used to recognize stress. The amplitude of harmonic sub-signals was negatively related to the number of harmonic decompositions $(\leq 3)$, indicating that the low-order harmonic amplitudes contain more energy information than high-order ones.

Variation laws of JM31, the variety of winter wheat sensitive to water content, were different from those of YF4 and JM38 in the RE region. Specifically, only harmonic amplitudes of JM31 show evident laws in the RE region. As a result, the harmonic amplitudes of JM31 in RE are listed in Table 3.

It can be seen in Table 3 that in the heading stage, $c 1, c 2$, and $\mathrm{c} 3$ were positively related to stress levels. In the flowering stage, the values of $\mathrm{c1}, \mathrm{c} 2$, and $\mathrm{c} 3$ tended to increase with an increase in stress level, except for $\mathrm{c} 1$ and $\mathrm{c} 2$ at ML10d and ML15d. In the filling stage, the values of $\mathrm{c} 1, \mathrm{c} 2$, and $\mathrm{c} 3$ tended to increase with increased stress, except for $\mathrm{c} 1$ and $\mathrm{c} 2$ at ML5d and ML10d. Therefore, only c3 was appropriate to assess waterlogging stress levels for JM31 in the RE region. Harmonic sub-signals of JM31 in the RE region are shown in Figure 8. 
Table 3. Harmonic amplitudes in RE region-JM31.

\begin{tabular}{ccccccccc}
\hline Growing Stage & Amplitude & CK & ML5d & ML10d & ML15d & SL5d & SL10d & SL15d \\
\hline \multirow{3}{*}{ Heading stage } & c1 & 1.5068 & 1.5530 & 1.5921 & 1.6173 & 1.6832 & 1.8298 \\
& c2 & 0.6916 & 0.7095 & 0.7252 & 0.7368 & 0.7716 & 0.8160 & 0.8719 \\
& c3 & 0.4426 & 0.4476 & 0.4555 & 0.4558 & 0.4855 & 0.5122 & 0.5547 \\
Flowering stage & c1 & 1.2615 & 1.2802 & 1.3234 & 1.2868 & 1.3238 & 1.3844 & 1.4141 \\
& c2 & 0.5659 & 0.5723 & 0.5979 & 0.5845 & 0.6037 & 0.6256 & 0.6310 \\
\multirow{2}{*}{ Filling stage } & c3 & 0.3582 & 0.3623 & 0.3713 & 0.3734 & 0.3872 & 0.3994 & 0.3996 \\
& c1 & 0.8124 & 0.9268 & 0.8735 & 0.8885 & 1.0403 & 1.2329 & 1.7480 \\
& c2 & 0.3956 & 0.4290 & 0.4174 & 0.4213 & 0.4978 & 0.5747 & 0.7937 \\
& c3 & 0.2431 & 0.2690 & 0.2756 & 0.2806 & 0.3301 & 0.3509 & 0.5014 \\
\hline
\end{tabular}
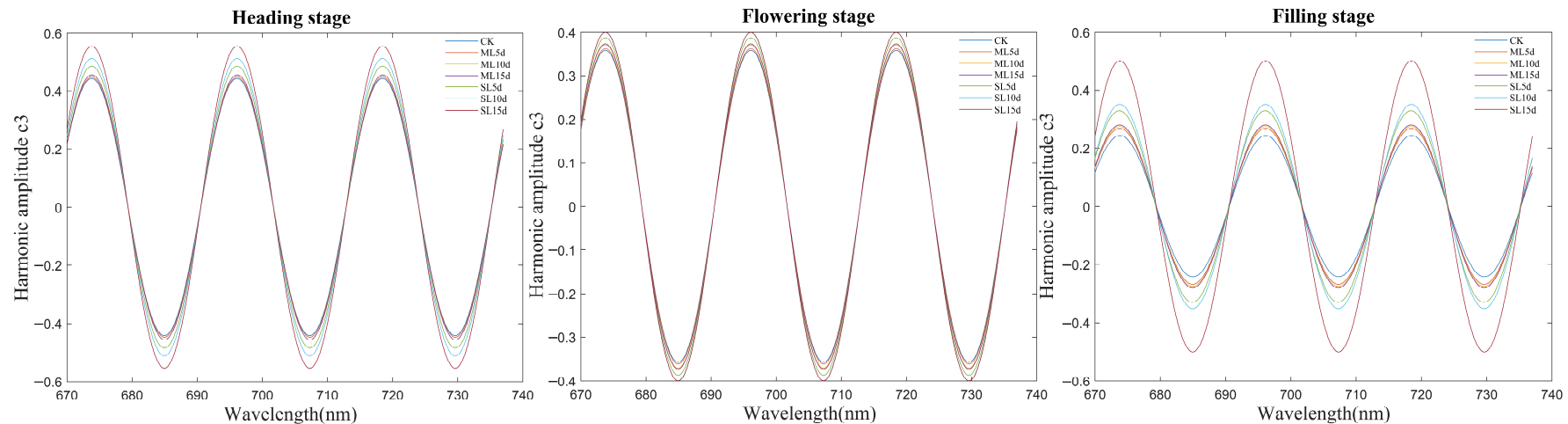

Figure 8. Harmonic sub-signals in the RE region-JM31.

Based on the above analysis:

(1) $\mathrm{c1}, \mathrm{c2}$, and $\mathrm{c} 3$ in the RW region can be used to recognize waterlogging stress levels in winter wheat, as their numerical values are positively related to stress levels. In the $\mathrm{RE}$ region, $\mathrm{c} 3$ can be used to recognize waterlogging stress levels in winter wheat that is sensitive to water. In the NIR region, the waterlogging stress levels of winter wheat cannot be recognized based on harmonic analysis. In short, c1, c2, and c3 in the RW region and c3 in the RE region can be used to assess waterlogging stress levels in winter wheat.

(2) $\mathrm{c} 1, \mathrm{c} 2$, and $\mathrm{c} 3$ in the RW region and $\mathrm{c} 3$ in the RE region show the following laws: CK $<$ ML5d $<$ ML10d $<$ ML15d $<$ SL5d $<$ SL10d $<$ SL15d or CK $<$ ML5d $<$ ML10d $<$ SL5d $<$ ML15d $<$ SL10d $<$ SL15d. This reflects the fact that short-term and middle-term mild waterlogging stress have less impact than severe waterlogging stress. The impact of longterm, mild waterlogging stress is similar to that of short-term, severe waterlogging stress.

(3) Harmonic sub-signals can recognize waterlogging stress levels in the heading, flowering, and filling stages of winter wheat, but cannot do so in the jointing and maturation stages. This might be due to the fact that the influences of waterlogging stress on crops are delayed in their presentation, and winter wheat is under stress at the jointing stage, so it shows no symptoms. In the maturation stage, the leaves of winter wheat begin to age, manifested by wilting and yellowing. As such, there are various influencing factors.

(4) Harmonic amplitudes of canopy spectra in the first three orders attenuate with the increase of decomposition times. In other words, low-order harmonic amplitudes contain more energy information than high-order harmonic amplitudes after harmonic decomposition.

\section{Discussion}

In this study, three varieties of winter wheat (YF4, JM31, and JM38) were chosen as the research subject. Gradient tests of waterlogging stress (CK, ML5d, ML10d, ML15d, SL5d, SL10d, and SL15d) were carried out in the jointing stage. Canopy hyperspectral imagery of winter wheat was collected in the jointing, heading, flowering, filling, and maturation 
stages, followed by classification and waveband screening. Evidence of waterlogging stress on winter wheat was assessed and examined based on harmonic analysis.

\subsection{Image Classification and Waveband Selection}

The RF algorithm demonstrated the best batch classification accuracy of hyperspectral imagery of potted winter wheat. This might be because hyperspectral data has high dimensions [44], and the RF algorithm can process high-dimensional data better than other classification algorithms without feature selection. Changes of leaf spectra are caused by changes in pigment, internal structure, and dry mass content [45]. It was concluded that the RW (640-680 nm), RE (670-737 nm), and NIR (750-900 nm) regions had the greatest capacity to recognize waterlogging stress (Table 2), which was consistent with the findings of previous studies [46]. In the RW region, spectral response features are mainly influenced by pigment content [20]. The RE region is sensitive to pigment and nutrients (mainly chlorophyll and nitrogen), and the influences of atmosphere and background can be decreased to a maximal extent $[47,48]$. Chlorophyll content has previously been used as an indicator of stress and growth conditions in vegetation. Stavros et al. [49] demonstrated that the RE region plays a key role in chlorophyll testing: CHLa is sensitive to $701 \mathrm{~nm}$ and $\mathrm{CHLb}$ is sensitive to $707 \mathrm{~nm}$. The NIR region is sensitive to internal leaf structure and dry mass content, including proteins, lignin, and cellulose [50].

\subsection{Harmonic Analysis}

\subsubsection{Pot Experiment}

Different levels of waterlogging stress influence the spectra of crops. Strong reflections of fresh leaf surfaces, cuticles of leaves, lamellar hair, and internal structures can also influence the spectra. It is difficult to monitor waterlogging stress level comprehensively and accurately from the spectral dimension alone. Harmonic analysis has a strong ability to analyze signals. Harmonic decomposition of spectra can decompose weak spectra distortion of waterlogging stress in the canopy spectra of winter wheat into low-order harmonics and decompose high-frequency noise into high-order harmonics. This is more beneficial for analyzing useful information in the spectral signals [33]. Changes or weak distortions in the canopy spectra of winter wheat reflect changes in the electromagnetic wave energies of the canopy spectra under different waterlogging stress levels. In this study, it was found that $\mathrm{c} 1, \mathrm{c} 2$, and $\mathrm{c} 3$ in the RW region can monitor the waterlogging stress levels of three varieties of winter wheat. In the RE region, c3 can be used to assess stress levels in the JM31 variety. This verifies that characteristic parameters of harmonic amplitudes can identify and distinguish weak spectral distortion in the RW and RE regions, especially in the RW region. After winter wheat suffers from waterlogging stress in the jointing stage, the heading stage, flowering stage, and filling stage are the best growth stages to assess stress levels based on harmonic analysis. No symptoms of waterlogging stress in the jointing stage may be caused by the hysteresis of influences. In the maturation stage, the leaves of winter wheat begin to age, manifested by wilting and yellowing. Moreover, there are many other influencing factors. Short-term and middle-term mild waterlogging stress has less impact than severe waterlogging stress. The impact of long-term, mild waterlogging stress is similar to that of short-term, severe waterlogging stress. This might be because under severe waterlogging stress, there exists oversaturated soil water and the slow diffusion of gases. Compared with mild waterlogging stress, $\mathrm{O}_{2}$ content decreases more quickly, thus resulting in a greater impact on plant growth. Long-term waterlogging stress causes oxygen toxicity in the root region of crops, thus resulting in energy shortage and toxin accumulation [51]. In addition, harmonic amplitudes of canopy spectra in the first three orders attenuate with the increase of decomposition times. In other words, the low-order harmonic amplitude contains more energy information than the high-order harmonic amplitude after harmonic decomposition. 


\subsubsection{Field Preliminary Verification}

Based on hyperspectral imagery of a pot experiment, this study monitored waterlogging stress levels in a frequency domain different from common spectral dimensions by using harmonic analysis. This provides an important method for the large-scale identification of environmental stress levels. To further deepen studies, applications of field-scale harmonic sub-signals can be discussed. Due to the scale problems of remote sensing, it is difficult to control and measure field waterlogging stress levels, and this was not the research focus of this study. The capacity of harmonic sub-signals in the RW region to assess waterlogging stress was discussed preliminarily based on UAV hyperspectral imagery. Hyperspectral imagery of winter wheat in the RW region was extracted, and each pixel point in the images was processed by three harmonic decompositions, resulting in the harmonic amplitudes c1, c2, and c3. These amplitudes were drawn in a diagram (Figure 9; blue box-control, red box-waterlogging stress). It demonstrates that there are obvious differences between control and stressed fields in term of harmonic amplitudes. Specifically, c1 from the control field was about $0.9 \times 10^{-3}-1.5 \times 10^{-3}$ and $1.6 \times 10^{-3}-3 \times 10^{-3}$ in the stressed field. $\mathrm{c} 2$ was about $0.4 \times 10^{-3}-0.63 \times 10^{-3}$ in the control field compared to $0.7 \times 10^{-3}-1.2 \times 10^{-3}$ in the stressed field. Finally, $\mathrm{c} 3$ was about $0.3 \times 10^{-3}-0.5 \times 10^{-3}$ in the control field and $0.6 \times 10^{-3}-0.9 \times 10^{-3}$ in the stressed field. Generally, field harmonic amplitudes were smaller compared to the potting harmonic amplitudes. However, c1, c2, and $\mathrm{c} 3$ from the stressed field were generally higher than those from the control field and attenuated with the increase of harmonic decomposition times $(\leq 3)$. This matches the conclusions gained from the canopy hyperspectral data of potted winter wheat. According to preliminary judgments, c1, c2, and c3 in the RW region can be used to assess waterlogging stress levels in fields of winter wheat. The results can preliminarily verify the effectiveness of the proposed method based on the pot experiment, and it can lay the foundations for large-scale monitoring. In the future, field experiments with multiple waterlogging stress gradients can be set up to deepen the research, with a view to perfecting the method and applying it to the actual monitoring of waterlogging stress levels.

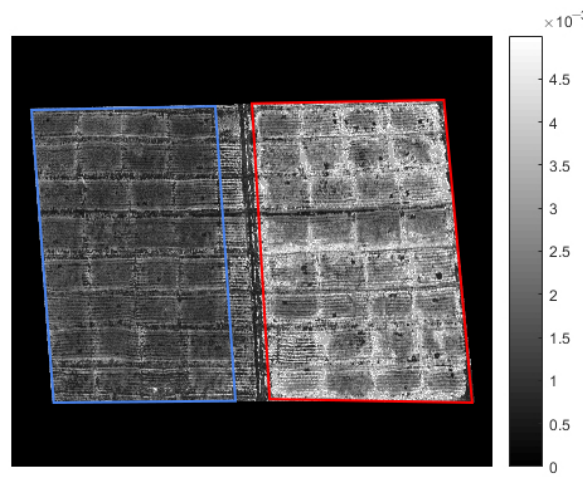

(a)

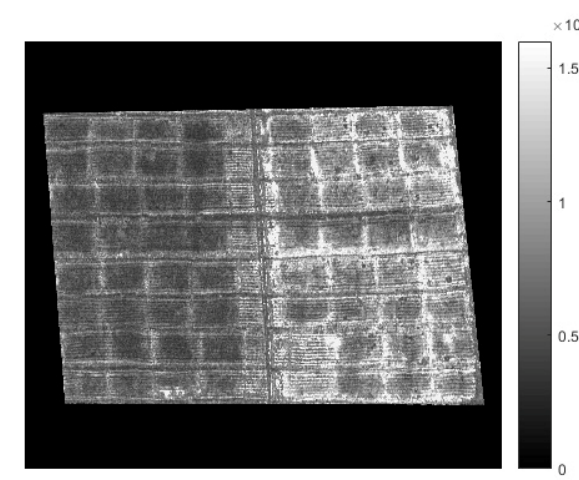

(b)

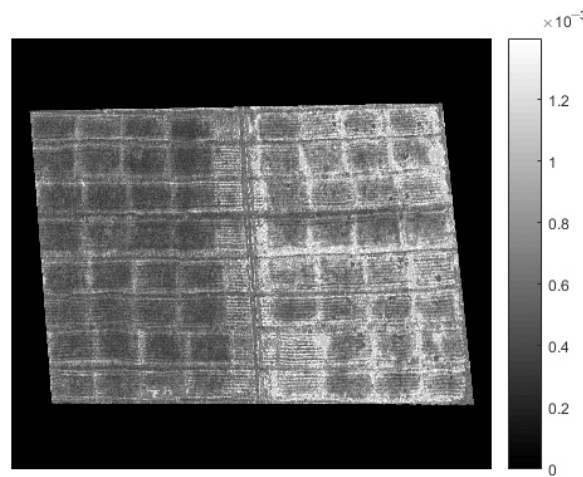

(c)

Figure 9. Harmonic amplitudes of c1, c2, c3 in the RW region. (a) c1; (b) c2; (c) c3.

\section{Conclusions}

In this study, a combined harmonic analysis method was proposed and preliminarily verified by using the hyperspectral imagery obtained through pot and field experiments in Yangzhou City, Jiangsu Province, China. This method could assess the waterlogging stress levels of winter wheat in the frequency domain different from the spectral dimension. Through the comparison of the batch classification accuracy of RF, SVM, and KNN, it was found that RF is the best batch classification method for hyperspectral imagery of potted winter wheat. Subsequently, the spectral data of winter wheat after classification was extracted, and a spectral distance $\left(d_{i}\right)$ was introduced for selecting the wavebands with strong recognition ability. It concluded that the RW (640-680 nm), RE (670-737 nm), and NIR (750-900 nm) regions could identify waterlogging stress levels well. Then, harmonic analy- 
sis was applied to assess the waterlogging stress levels of winter wheat in the frequency domain. This demonstrated that $c 1, c 2$, and $c 3$ in the RW region were the best indexes to use in the identification of waterlogging stress levels in winter wheat. Moreover, $\mathrm{c} 3$ in the RE region was appropriate for evaluating stress levels in the JM31 variety of wheat. After winter wheat suffered from waterlogging stress during jointing, the heading, flowering, and filling stages were the best growth intervals during which to assess stress levels based on harmonic analysis. Moreover, a preliminary field experiment on the ability of harmonic amplitude in the RW region to assess waterlogging stress was carried out. Preliminarily, it was determined that $\mathrm{c} 1, \mathrm{c} 2$, and $\mathrm{c} 3$ in the RW region can assess waterlogging stress in fields of winter wheat. This study provides a new method for assessing waterlogging stress levels. It has important theoretical significance and practical application values for the accurate control of waterlogging stress. It can be further verified and applied to studies on other types of environmental stress levels such as drought stress, freezing stress, and high-temperature stress levels.

Author Contributions: Conceptualization, methodology, validation, data curation, writing-original draft preparation, visualization, F.Y. and Q.W.; conceptualization, writing-review and editing, funding acquisition, S.L. (Shijuan Li); funding acquisition, resources, S.L. (Shengping Liu); resources, supervision, T.L. All authors have read and agreed to the published version of the manuscript.

Funding: This research was funded by the National Key Research and Development Program of China (No. 2016YFD0200600, No. 2016YFD0200601).

Institutional Review Board Statement: Not applicable.

Informed Consent Statement: Not applicable.

Data Availability Statement: The data presented in this study are available on request from the corresponding author. The data are not publicly available due to that the follow-up related work has not been completed.

Acknowledgments: The authors wish to express heartfelt thanks to Chengming Sun for his kind help, and to Tianle Yang, Zhaosheng Yao, Ping Zhou, Yunian Sun for data acquisition work.

Conflicts of Interest: The authors declare no conflict of interest.

\section{References}

1. Bastawesy, M.E.; Ali, R.R.; Harbi, K.A.; Faid, A. Impact of the geomorphology and soil management on the development of waterlogging in closed drainage basins of Egypt and Saudi Arabia. Environ. Earth Sci. 2012, 68, 1271-1283. [CrossRef]

2. Gebrehiwot, K.A. A review on waterlogging, salinization and drainage in Ethiopian irrigated agriculture. Sustain. Water Resour. Manag. 2018, 4, 55-62. [CrossRef]

3. Radda, I.A.; Kumar, B.M.; Pathak, P. Land Degradation in Bihar, India: An Assessment Using Rain-Use Efficiency and Residual Trend Analysis. Agric. Res. 2021, 10, 434-447. [CrossRef]

4. Pandey, A.C.; Singh, S.K.; Nathawat, M.S. Waterlogging and flood hazards vulnerability and risk assessment in Indo Gangetic plain. Nat. Hazards 2010, 55, 273-289. [CrossRef]

5. Ding, J.; Liang, P.; Wu, P.; Zhu, M.; Li, C.; Zhu, X.; Gao, D.; Chen, Y.; Guo, W. Effects of waterlogging on grain yield and associated traits of historic wheat cultivars in the middle and lower reaches of the Yangtze River, China. Field Crop. Res. 2020, $246,107695$. [CrossRef]

6. Yang, H.; Zhai, S.; Li, Y.; Zhou, J.; He, R.; Liu, J.; Xue, Y.; Meng, Y. Waterlogging reduction and wheat yield increase through long-term ditch-buried straw return in a rice-wheat rotation system. Field Crop. Res. 2017, 209, 189-197. [CrossRef]

7. Zúñiga-Feest, A.; Bustos-Salazar, A.; Alves, F.; Martinez, V.; Smith-Ramírez, C. Physiological and morphological responses to permanent and intermittent waterlogging in seedlings of four evergreen trees of temperate swamp forests. Tree Physiol. 2017, 37, 779-789. [CrossRef]

8. Romina, P.; Abeledo, L.G.; Miralles, D.J. Physiological traits associated with reductions in grain number in wheat and barley under waterlogging. Plant Soil 2018, 429, 469-481.

9. Wu, X.; Tang, Y.; Li, C.; McHugh, A.; Li, Z.; Wu, C. Individual and combined effects of soil waterlogging and compaction on physiological characteristics of wheat in southwestern China. Field Crop. Res. 2018, 215, 163-172. [CrossRef]

10. Das, P.K.; Choudhary, K.K.; Laxman, B.; Rao, S.K.; Seshasai, M. A modified linear extrapolation approach towards red edge position detection and stress monitoring of wheat crop using hyperspectral data. Int. J. Remote Sens. 2014, 35, 1432-1449. [CrossRef] 
11. Hede, A.N.H.; Kashiwaya, K.; Koike, K.; Sakurai, S. A new vegetation index for detecting vegetation anomalies due to mineral deposits with application to a tropical forest area. Remote Sens. Environ. 2015, 171, 83-97. [CrossRef]

12. Tianjiao, L.; Xiangnan, L.; Meiling, L.; Ling, W. Evaluating heavy metal stress levels in rice based on remote sensing phenology. Sensors 2018, 18, 860.

13. Zhang, B.; Liu, X.; Liu, M.; Wang, D. Thermal infrared imaging of the variability of canopy-air temperature difference distribution for heavy metal stress levels discrimination in rice. J. Appl. Remote Sens. 2017, 11, 26036. [CrossRef]

14. Li, X.; Li, L.; Liu, X. Collaborative inversion heavy metal stress in rice by using two-dimensional spectral feature space based on HJ-1 A HSI and radarsat-2 SAR remote sensing data. Int. J. Appl. Earth Obs. Geoinf. 2019, 78, 39-52. [CrossRef]

15. Dhau, I.; Adam, E.; Mutanga, O.; Ayisi, K.K. Detecting the severity of maize streak virus infestations in maize crop using in situ hyperspectral data. Trans. R. Soc. S. Afr. 2018, 73, 8-15. [CrossRef]

16. Qiao, H.; Jiao, H.; Shi, Y.; Shi, L.; Guo, W.; Ma, X. Using Hyperspectral Remote Sensing Identification of Wheat Take-All Based on SVM. Secur. Educ. Crit. Infrastruct. 2015, 452, 23-30.

17. Jin, N.; Huang, W.; Ren, Y.; Luo, J.; Wu, Y.; Jing, Y.; Wang, D. Hyperspectral identification of cotton verticillium disease severity. Opt.-Int. J. Light Electron Opt. 2012, 124, 2569-2573. [CrossRef]

18. Huang, L.; Zhang, H.; Ding, W.; Huang, W.; Hu, T.; Zhao, J. Monitoring of Wheat Scab Using the Specific Spectral Index from ASD Hyperspectral Dataset. J. Spectrosc. 2019, 2019, 9153195. [CrossRef]

19. Zhang, J.; Dai, L.; Cheng, F. Identification of Corn Seeds with Different Freezing Damage Degree Based on Hyperspectral Reflectance Imaging and Deep Learning Method. Food Anal. Methods 2021, 14, 389-400. [CrossRef]

20. Liang, L.; Xiang, L.; Qin, S.; Jia, R.; Hui, L. Diagnosis the dust stress of wheat leaves with hyperspectral indices and random forest algorithm. In Proceedings of the IGARSS 2016-2016 IEEE International Geoscience and Remote Sensing Symposium, Beijing, China, 11-15 July 2016; pp. 6385-6388.

21. Yang, F.-F.; Liu, T.; Wang, Q.-Y.; DU, M.-Z.; Yang, T.-L.; Liu, D.-Z.; Li, S.-J.; Liu, S.-P. Rapid determination of leaf water content for monitoring waterlogging in winter wheat based on hyperspectral parameters. J. Integr. Agric. 2021, 20, 2613-2626. [CrossRef]

22. Zhao, J.; Pan, F.; Li, Z.; Lan, Y.; Lu, L.; Yang, D.; Wen, Y. Detection of cotton waterlogging stress based on hyperspectral images and convolutional neural network. Int. J. Agric. Biol. Eng. 2021, 14, 167-174. [CrossRef]

23. Xiong, Q.-X.; Wang, X.-L.; Wang, Y.-N. Spectral Characteristics Analysis of Wheat Damaged by Subsurface Waterlogging. Spectrosc. Spect. Anal. 2016, 36, 2558-2561.

24. Jiang, J.; Steven, M.D.; He, R.; Chen, Y.; Du, P.; Guo, H. Identifying the spectral responses of several plant species under CO2 leakage and waterlogging stresses. Int. J. Greenh. Gas Control. 2015, 37, 1-11. [CrossRef]

25. Emengini, E.J.; Blackburn, G.A.; Theobald, J.C. Discrimination of plant stress caused by oil pollution and waterlogging using hyperspectral and thermal remote sensing. J. Appl. Remote Sens. 2013, 7, 073476. [CrossRef]

26. Xia, J.; Cao, H.; Yang, Y.; Zhang, W.; Wan, Q.; Xu, L.; Ge, D.; Zhang, W.; Ke, Y.; Huang, B. Detection of waterlogging stress based on hyperspectral images of oilseed rape leaves (Brassica napus L.). Comput. Electron. Agric. 2019, 159, 59-68. [CrossRef]

27. Yang, F.; Liu, S.; Zhu, Y.; Li, S. Identification and level discrimination of waterlogging stress in winter wheat using hyperspectral remote sensing. Smart Agric. 2021, 3, 35-44.

28. Peng, Y.-Q.; Zhu, J.; Li, W.-J.; Gao, W.; Shen, R.-Y.; Meng, L.-J. Effects of grafting on root growth, anaerobic respiration enzyme activity and aerenchyma of bitter melon under waterlogging stress. Sci. Hortic. 2020, 261, 108977. [CrossRef]

29. Zouari, M.; Bloem, E.; Souguir, D.; Schnug, E.; Hanchi, B.; Hachicha, M. Evaluation of selected plant species under drained saline and waterlogged conditions in pots when irrigated with treated wastewater. Water Environ. J. 2020, 34, 551-560. [CrossRef]

30. Chen, L.; Zheng, D.; Chen, S.; Han, B. Method Based on Sparse Signal Decomposition for Harmonic and Inter-harmonic Analysis of Power System. J. Electr. Eng. Technol. 2017, 12, 559-568. [CrossRef]

31. Xue, Z.; Du, P.; Su, H. Harmonic Analysis for Hyperspectral Image Classification Integrated with PSO Optimized SVM. IEEE J. Sel. Top. Appl. Earth Obs. Remote Sens. 2014, 7, 2131-2146. [CrossRef]

32. Yang, G.; Shen, H.; Zhang, L.; He, Z.; Li, X. A Moving Weighted Harmonic Analysis Method for Reconstructing High-Quality SPOT VEGETATION NDVI Time-Series Data. IEEE Trans. Geosci. Remote Sens. 2015, 53, 6008-6021. [CrossRef]

33. Jakubauskas, M.E.; Legates, D.R.; Kastens, J.H. Crop identification using harmonic analysis of time-series AVHRR NDVI data. Comput. Electron. Agric. 2002, 37, 127-139. [CrossRef]

34. Cao, X.; Li, R.; Ge, Y.; Wu, B.; Jiao, L. Densely connected deep random forest for hyperspectral imagery classification. Int. J. Remote Sens. 2018, 40, 3606-3622. [CrossRef]

35. Ji, Y.; Sun, L.; Li, Y.; Li, J.; Liu, S.; Xie, X.; Xu, Y. Non-destructive classification of defective potatoes based on hyperspectral imaging and support vector machine. Infrared Phys. Technol. 2019, 99, 71-79. [CrossRef]

36. Cortes, C.; Vapnik, V.N. Support-vector networks. Mach. Learn. 1995, 20, 273-297. [CrossRef]

37. Bo, C.; Lu, H.; Wang, N. Spectral-spatial K-Nearest Neighbor approach for hyperspectral image classification. Multimed. Tools Appl. 2018, 77, 10419-10436. [CrossRef]

38. Tong, Q.X.; Zhang, B.; Zheng, L.F. Hyperspectral Remote Sensing: Principles, Techniques, and Applications; Higher Education Press: Beijing, China, 2006.

39. Wang, R.; Gamon, J.A.; Cavender-Bares, J.; Townsend, P.A.; Zygielbaum, A.I. The spatial sensitivity of the spectral diversitybiodiversity relationship: An experimental test in a prairie grassland. Ecol. Appl. 2018, 28, 541-556. [CrossRef] 
40. Yang, K.; Xue, Z.; Jia, T.; Zhang, T.; Wang, L. A harmonic analysis model of small target detection of hyperspectral imagery. Acta Geod. Cartogr. Sin. 2013, 42, 34-43.

41. Guo, H.; Yang, K.M.; Zhang, W.W.; Liu, C.; Xia, T. Spectra recognition of corn pollution degree under copper and lead lon stress. Guangpuxue Yu Guangpu Fenxi/Spectrosc. Spectr. Anal. 2018, 38, 212-217.

42. Geng, X.; Sun, K.; Ji, L. Band selection for target detection in hyperspectral imagery using sparse CEM. Remote Sens. Lett. 2014, 5, 1022-1031. [CrossRef]

43. Sun, K.; Geng, X.; Ji, L. Exemplar Component Analysis: A Fast Band Selection Method for Hyperspectral Imagery. IEEE Geosci. Remote Sens. Lett. 2014, 12, 998-1002.

44. Yu, W.; Zhang, M.; Shen, Y. Learning a local manifold representation based on improved neighborhood rough set and LLE for hyperspectral dimensionality reduction. Signal Process. 2019, 164, 20-29. [CrossRef]

45. Vilfan, N.; van der Tol, C.; Yang, P.; Wyber, R.; Malenovský, Z.; Robinson, S.; Verhoef, W. Extending Fluspect to simulate xanthophyll driven leaf reflectance dynamics. Remote Sens. Environ. 2018, 211, 345-356. [CrossRef]

46. Qian, X.; Qiu, S.; Liang, L.; Zhang, L.; Wang, S. Urban green vegetation stress conditions diagnosis based on hyperspectral database-A case study of Xuzhou. In Proceedings of the 2014 Third International Conference on Agro-Geoinformatics, Beijing, China, 11-14 August 2014; pp. 1-4.

47. Ramoelo, A.; Skidmore, A.; Cho, M.A.; Schlerf, M.; Mathieu, R.; Heitkonig, I. Regional estimation of savanna grass nitrogen using the red-edge band of the spaceborne RapidEye sensor. Int. J. Appl. Earth Obs. Geoinf. 2012, 19, 151-162. [CrossRef]

48. Ramoelo, A.; Dzikiti, S.; van Deventer, H.; Maherry, A.; Cho, M.A.; Gush, M. Potential to monitor plant stress using remote sensing tools. J. Arid. Environ. 2015, 113, 134-144. [CrossRef]

49. Stagakis, S.; Markos, N.; Sykioti, O.; Kyparissis, A. Monitoring canopy biophysical and biochemical parameters in ecosystem scale using satellite hyperspectral imagery: An application on a Phlomis fruticosa Mediterranean ecosystem using multiangular CHRIS/PROBA observations. Remote Sens. Environ. 2010, 114, 977-994. [CrossRef]

50. Liu, L.-Y.; Huang, W.-J.; Pu, R.-L.; Wang, J.-H. Detection of Internal Leaf Structure Deterioration Using a New Spectral Ratio Index in the Near-Infrared Shoulder Region. J. Integr. Agric. 2014, 13, 760-769. [CrossRef]

51. Liu, W.; Huang, J.; Wei, C.; Wang, X.; Mansaray, L.R.; Han, J.; Zhang, D.; Chen, Y. Mapping water-logging damage on winter wheat at parcel level using high spatial resolution satellite data. ISPRS J. Photogramm. Remote Sens. 2018, 142, 243-256. [CrossRef] 